\title{
A Sealed-Bid Auction that Matches the English Auction ${ }^{1}$
}

\author{
MOTTY PERRY \\ Dept. of Economics \\ Hebrew University of Jerusalem \\ Mount Scopus \\ Jerusalem 91905 \\ Israel \\ motty@sunset.huji.ac.il
}

\author{
ELMAR WOLFSTETTER \\ Dept. of Economics \\ Humboldt-Universität zu Berlin \\ Spandauer Str. 1 \\ 10178 Berlin \\ Germany \\ wolf@wiwi.hu-berlin.de
}

\author{
SHMUEL ZAMIR \\ Dept. of Statistics \\ Hebrew University of Jerusalem \\ Mount Scopus \\ Jerusalem 91905 \\ Israel \\ zamir@math.huji.ac.il.
}

JUNE 1998

\begin{abstract}
${ }^{1}$ Financial support was received by the Center for Rationality, Hebrew University in Jerusalem, and the Deutsche Forschungsgemeinschaft, SFB 373 ("Quantifikation und Simulation Ökonomischer Prozesse"), Humboldt-Universität zu Berlin. Partial support to Perry and Zamir was provided by the Koret Foundation. We are indebted to Todd Kaplan for very useful discussions.
\end{abstract}




\begin{abstract}
This paper analyzes a two-stage sealed-bid auction that is frequently employed in privatization, takeover, and merger and acquisition contests. This auction format yields the same expected revenue as the open ascending (English) auction, yet is less susceptible to preemptive bidding and collusion.
\end{abstract}

JEL classifications: D44 (Auctions)

Keywords: Auctions 


\section{Introduction}

An important issue in the theory of auction design is whether one should employ an open, ascending bid (English) or a sealed-bid auction. The open format is generally advised on the ground that it furnishes bidders with valuable information. This reduces the winner's curse, which contributes to more aggressive bidding, to the benefit of the seller.

In their seminal contribution, Milgrom and Weber (1982) showed that the open-bid ascending auction yields higher revenues than the sealedbid (second or first price) auctions. However, the exchange of information in the course of an English auction also has a drawback: It may invite preemptive or jump bidding, ${ }^{1}$ and it may be misused by bidders to communicate and enforce collusion.

Casual observation of real world auctions reveals that in many cases the seller employs a different two-stage sealed-bid auction that does not fit the usual distinction of auction formats. For example, in Italy, the formerly state owned industrial conglomerate ENI was privatized using such a two-stage procedure. In the first round bidders submitted sealed-bids and reorganization plans. Then, the auctioneer screened out the lowest bidders and finalized the sale in the second round, in a sealed-bid auction, with the proviso that bids could not be lowered. ${ }^{2}$ A similar tworound sealed-bid format is frequently observed in takeover and merger and acquisition contests. Indeed, if one talks to investment bankers, they describe this two-stage format as the standard procedure, and they justify it with the high cost of bidding and the fact that preemptive bidding would be a serious problem in an open, ascending auction.

The purpose of this note is to rationalize this type of auctions. We study the following two-stage auction in the context of the Milgrom and Weber model: In the first stage, all agents simultaneously submit sealed bids. Only the two highest bidders continue to the second stage. All bids that fail to pass to the second stage are publicly revealed. In the second stage, the two remaining bidders play a sealed-bid second price auction where each bidder is bounded by his or her own first round bid.

\footnotetext{
${ }^{1}$ A "preemptive bid", also called a "jump bid”, is a high initial bid, with the intention of encouraging others to quit the auction. For explanations of jump bidding see Fishman (1988).

${ }^{2}$ For a detailed account of the breaking-up of ENI (Italian Oil and Energy Corporation) see Caffarelli (1997). A peculiar feature of the ENI auction was that all bids (not just the losing bids) were made known. Therefore, the second-round auction was potentially an auction in which the ranking of valuations was common knowledge. Such auctions are analyzed in Landsberger, Rubinstein, Wolfstetter and Zamir (1997).
} 
We show that this design, which minimizes the opportunity of bidders to send signals back and forth via their bids, yields an expected revenue as high as the ascending open-bid (English) auction.

\section{The Model}

Consider the well-known symmetric affiliated values model by Milgrom and Weber (1982), which includes the symmetric private values and the symmetric common value model as special cases.

One indivisible unit of a certain good is auctioned to $n \geq 2$ risk neutral bidders. Prior to the auction, each bidder receives a signal or value estimate $X_{i}$ of the object for sale. The vector of signals $X=\left(X_{i}\right)_{i \in N}$ is drawn from a continuous and symmetric joint distribution $F$ with support $[0,1]^{n}$. Bidders' valuations are an identical function of their own signal and the set of rivals' signals $X_{-i}:=\left(X_{j}\right)_{j \neq i}$

$$
V\left(X_{i}, X_{-i}\right) .
$$

$V$ is nonnegative, continuous, and strictly increasing in each of its variables and symmetric in $\left\{X_{j}\right\}_{j \neq i}$.

The auction rules have two rounds, as follows:

RounD 1 Each bidder, after observing his private signal, submits a closedsealed bid. The two highest bidders are allowed to continue; for all others the game is over. Ties are resolved by an equal probability rule.

Round 2 The auctioneer publicly announces the bids rejected in round 1 and runs a second-price auction among the two remaining bidders. Bidders must bid at least their own bid from round 1 and no one is allowed to withdraw from bidding. Again, ties are broken by an equal probability rule.

We denote by $b(x)$ the 1 -st round bid function, defined on $[0,1]$, and by $\beta(x, B)$ the $2-$ nd round bid, where $x$ is the bidder's signal and $B$ the set of rejected 1-st round bids that were announced after the 1-st round.

The joint probability distribution $F$ is symmetric, and the random variables $X_{1}, X_{2}, \ldots, X_{n}$ are affiliated, as defined in Milgrom and Weber (1982). For technical convenience we assume that the joint distribution of signals has a density $f$ satisfying $0<\underline{c}<f(x)<\bar{c}<+\infty$ on $[0,1]^{n}$. 
We denote the highest, the second highest etc. signal received by rival bidders by the order statistics $Y_{1}, Y_{2}, \ldots, Y_{n-1}$ respectively, and let $Z$ be the vector of order statistics of the lowest $n-2$ rival bidders' signals, $Z=\left(Y_{2}, . ., Y_{n-1}\right)$.

Finally, we restrict the analysis to symmetric equilibria with (strictly) increasing bid functions. In this case, the signals of the rejected bidders can be deduced from the rejected bids $B$. In view of the symmetry of $V$ in the coordinates of $X_{-i}$, the dependence of all relevant functions on $B$ is only through $Y_{1}$ and the vector of order statistics $Z$. Therefore, we will always write $\beta(x, Z)$ and $V\left(x, Y_{1}, Z\right)$.

\section{Equilibrium}

In this section we show that all Nash equilibria yield the same equilibrium outcome as the open ascending (English) auction. Among these equilibria there is a unique strict equilibrium which is also the unique (trembling hand) perfect Nash equilibrium.

Let $b$ be a strictly increasing function on $[0,1]$ and consider the following " $b$-restricted auction" which is equivalent to the original auction except that bidders are restricted to play the strategy $b$ in the first round.

PROPOSITION 1 (SECOND-Round BIDDING)

$$
\beta(x, z):=\max \{b(x), V(x, x, z)\}
$$

is the unique symmetric equilibrium of the b-restricted auction (in the class of pure monotone bid strategies).

PROOF In the absence of the constraint on 2-nd round bidding this is a straightforward adaptation of Milgrom and Weber (1982), Sect. 5. Recall that only two bidders are allowed to participate in the second round, and note that in this case a bidder who plays this strategy would never benefit from bidding differently even if he knew the rival's signal. In the presence of the constraint it may happen that a first-round winner with a signal $x$ could benefit from lowering his bid so that he loses the auction when his rival is constrained by his 1-st round bid. However, as one can see easily, this can happen only if the winning bidder cannot lower his bid due to his own constraint (note that the 1-st round winning bidder's constraint exceeds that of his rival, due to the monotonicity of $b$ ).

In view of Proposition 1 we refer to $b$ as a strategy with the understanding that 2 -nd round bidding is according to (1). 
LEMMA 1 Consider a bidder with a signal $X=x$. Suppose rival bidders bid according to the same strict monotone increasing bid function $b$. If $\tilde{b}(x)$ is a (strictly) better response than $b(x)$, then $\tilde{b}(x)<b(x)$.

PROOF Suppose instead of bidding $b(x)$, a bidder with signal $x$ bids $\tilde{b}(x)>b(x)$ while all others are bidding according to $b$. This bid can make a difference only if he thus wins the auction, but would have lost it while bidding $b(x)$. Therefore, it must be true that $x<Y_{1}$. Thus, when winning the auction, the bidder pays $V\left(Y_{1}, Y_{1}, z\right)$ which is greater than his own valuation $V\left(x, Y_{1}, z\right)$. Hence, if everyone else bids according to strategy $b$, it never pays to bid higher than $b(x)$.

LEMMA 2 Any strict monotone increasing $b$ such that $b(x) \leq V(x, x, 0)$ is a symmetric Nash equilibrium.

PROOF First note that when all bidders bid according to $b(x) \leq V(x, x, 0)$, a bidder with signal $x$ who is allowed to participate in the 2-nd round never regrets bidding $b(x)$ in the 1-st round, even if he knew rivals' signals, since $V(x, x, z) \geq V(x, x, 0) \geq b(x)$. By Lemma 1 any profitable deviation $\tilde{b}(x)$ from $b(x)$ must satisfy $\tilde{b}(x)<b(x)$. Such a deviation makes a difference only in states where $x>Y_{1}$ and $\tilde{b}(x)<b\left(Y_{2}\right)$. In such a state, by bidding $\tilde{b}(x)$ he does not qualify for the 2-nd round and thus foregoes a net profit of $V\left(x, Y_{1}, z\right)-V\left(Y_{1}, Y_{1}, z\right)>0$, which he would have as a winner by playing $b(x)$.

LEMMA 3 Assume $b$ is strict monotone increasing with $b(x) \leq V(x, x, 0)$, $\forall x$. If all rival players bid according to $b$ then $V(x, x, 0)$ is a best reply while any $\tilde{b}(x)<b(x)$ is not a best reply.

Proof The first part is true because $\forall z, V(x, x, 0) \leq V(x, x, z)$; hence, bidding $V(x, x, 0)$ does not constrain 2-nd round bidding. On the other hand, when bidding $\tilde{b}(x)<b(x)$, while all rivals bid according to $b$, there is a positive probability that the signal $x$ is the highest, but the bidder will not get into the 2-nd round and hence foregoes a positive profit.

COROLlaRY $1 b^{*}(x):=V(x, x, 0)$ is a symmetric strict equilibrium and any equilibrium $b \nsupseteq b^{*}$ is not strict.

Proof We already know that $b^{*}$ is an equilibrium. By Lemma 3, no deviation $b(x)<b^{*}(x)$ is a best reply to $b^{*}$. It remains to be shown that no deviation $b(x)>b^{*}(x)$ is a best reply to $b^{*}$. In fact, by Lemma 1 , 
$b(x)$ is not a strictly better response than $b^{*}(x)$. To see that it cannot be a best reply to $b^{*}$, observe that for sufficiently small $z$, there is $y_{1}>x$ s.t. $b(x)>V\left(y_{1}, y_{1}, z\right)>V\left(x, y_{1}, z\right)$. In such an event (which is of positive probability), the player will win the object paying $V\left(y_{1}, y_{1}, z\right)$, which is more than what it is worth to him, $V\left(x, y_{1}, z\right)$. Thus, $b(x)$ is a strictly worse reply than $b^{*}(x)$, completing the proof that $b^{*}$ is a strict equilibrium. Any equilibrium $b \lesseqgtr b^{*}$ is not strict, since by Lemma $3, b^{*}$ is also a best reply to $b$.

COROLLARY 2 The equilibrium $b^{*}$ is (trembling hand) perfect, while any other equilibrium $b \lesseqgtr b^{*}$ is not perfect.

Proof Consider an equilibrium $b \lesseqgtr b^{*}$. When in the perturbed game all players use a uniformly perturbed strategy (tremble) around $b$, then a best reply is $b^{*}(x)$ which is strictly better than $b(x)$ (since with positive probability $x>y_{1}$ and $b(x)<b\left(y_{2}\right)<b^{*}(x)$ in which case $b^{*}(x)$ is strictly better than $b(x)$.) Hence, as the perturbation tends to 0 , the limiting best reply is $b^{*}(x)$.

THEOREM 1 (FIRST-ROUND BIDDING) There is no Nash equilibrium $b(x) \rightrightarrows$ $b^{*}(x)$ and $b^{*}(x):=V(x, x, 0)$ is the only (trembling hand) perfect equilibrium.

PROOF Suppose there exists another function $b(x) \rightrightarrows b^{*}(x)$ that generates an equilibrium profile. We then show that, unless $b(x)=b^{*}(x)$, the bid function $b$ is not a best reply assuming all others play $b$. This proves that there is no equilibrium above $b^{*}$ from which the last assertion follows by Corollary 2 .

To see the heuristics of the main argument, let $x_{0}>0$ and $b\left(x_{0}\right)=$ $b^{*}\left(x_{0}\right)+\delta\left(x_{0}\right)$ where $\delta\left(x_{0}\right)>0$. If the bidder with the signal $x_{0}$ bids in the first round $b\left(x_{0}-\varepsilon\right)$ rather than $b\left(x_{0}\right)$ he increases his payoff in the event $Y_{2}<x_{0}-\varepsilon<Y_{1}<x_{0}$ in the order of $\delta\left(x_{0}\right) \varepsilon$, and decreases it in the event $x_{0}-\varepsilon<Y_{2}<Y_{1}<x_{0}$ in the order of $\varepsilon^{2}$. Thus, if $\delta\left(x_{0}\right)>0$, for sufficiently small $\varepsilon$ the payoff increases.

The full details of the proof are spelled out in the Appendix.

Recall that in the equilibrium of the English auction, once only two bidders are active, they play as in a second-price auction after the $n-2$ lowest signals have become known (see Milgrom and Weber (1982)). In equilibrium this is precisely the situation in the second-round of our game. Therefore, we conclude: 
THEOREM 2 (REVENUE EQUIVALENCE) The strict and perfect equilibrium $b^{*}$ (as well as each other non-strict equilibrium) is revenue equivalent to the corresponding English auction.

\section{Conclusion}

We have analyzed a simple two-stage sealed-bid auction and showed that it has a unique symmetric strong equilibrium that is payoff equivalent to the symmetric equilibrium of the English auction. We now briefly summarize and discuss the merits of this auction rule.

It is useful to compare it to two revenue-equivalent mechanisms: 1) the open ascending (English) auction, and 2) the associated direct incentive compatible mechanism. ${ }^{3}$ Of course, the latter exists, by the revelation principle, and it can be implemented in one stage and as a closed-seal bid. We evaluate these mechanisms according to simplicity of rules, ease of implementation, revenue, and susceptibility to collusion and jump bidding.

The rules of the open ascending (English) auction are fairly simple, but implementation is complex and costly, and it is vulnerable to both collusion and jump bidding. The associated direct incentive compatible mechanism is easy to implement, but the rules are complicated and perhaps too difficult for buyers to understand. The proposed two-stage auction combines the best of 1) and 2). Indeed, the rules of the twostage auction are at least as simple as those of the English auction, and their implementation is as simple as that of the direct incentive compatible mechanism. In addition, it is less susceptible to collusion and jump bidding.

In view of these results it is not surprising that the two-stage sealedbid auction format is commonly employed in privatization, takeover, and merger and acquisition contests.

\section{Appendix}

Proof OF THEOREM 1 Assume $b(x):=b^{*}(x)+\delta(x)$ with $\delta(x) \geq 0, \forall x$, is an increasing function that generates a symmetric equilibrium profile.

\footnotetext{
${ }^{3}$ Other standard auctions like first-price and Dutch auctions are ignored, since they are less profitable for the seller in any case (see Milgrom and Weber (1982)).
} 
We show that $b(x)$ is not a best reply to $b(x)$ (used by all other players) unless $\delta(x) \equiv 0$.

Suppose that for some $x_{0}>0$ one has $\delta\left(x_{0}\right)>0$. Since $b$ is strictly monotone it has at most countably many discontinuities. Therefore, we can assume that there is such an $x_{0}$ where $b$ is continuous.

Consider a bidder with signal $x_{0}$ and assume all other bidders play the strategy profile generated by $b$. Denote by $\pi\left(x_{0}, x_{0}\right)$ the payoff of that bidder if he follows the same strategy and by $\pi\left(x_{0}, x_{0}-\varepsilon\right)$ his payoff if he deviates to $b\left(x_{0}-\varepsilon\right)$ (followed by the $\beta$ continuation). We shall show that if $\delta\left(x_{0}\right)>0$, then for sufficiently small $\varepsilon$ one has $\pi\left(x_{0}, x_{0}-\varepsilon\right)>$ $\pi\left(x_{0}, x_{0}\right)$.

Note first that if $x_{0}$ is not the highest signal, bidding $b\left(x_{0}-\varepsilon\right)$ yields the same payoff as bidding $b\left(x_{0}\right)$ because that bidder loses the auction in both cases. Similarly, if $Y_{2}<Y_{1}<x_{0}-\varepsilon<x_{0}$, then bidding $b\left(x_{0}\right)$ also yields the same payoff as $b\left(x_{0}-\varepsilon\right)$ because in both cases the bidder wins the auction and pays the bid of the player with signal $Y_{1}$. Therefore, we only need to evaluate the change in payoff in the following two events

$$
\begin{aligned}
& A_{1}:=\left\{Y_{1}, Y_{2} \mid Y_{2}<x_{0}-\varepsilon<Y_{1}<x_{0}\right\} \\
& A_{2}:=\left\{Y_{1}, Y_{2} \mid x_{0}-\varepsilon<Y_{2}<Y_{1}<x_{0}\right\} .
\end{aligned}
$$

In the event $A_{1}$ the bidder enters the 2-nd round both with $b\left(x_{0}\right)$ and with $b\left(x_{0}-\varepsilon\right)$. However, bidding $b\left(x_{0}-\varepsilon\right)$ makes him less constrained in the 2-nd round bidding, and hence yields a (conditional) payoff at least as large as that from bidding $b\left(x_{0}\right)$. To compute the increment in payoff in this event define

$$
\begin{aligned}
\mathcal{Z}\left(x_{0}\right):= & \left\{Z \mid E_{\left\{Y_{1} \mid Y_{1}<x_{0}\right\}}\left(V\left(x_{0}, Y_{1}, Z\right)\right.\right. \\
& \left.\left.-\max \left\{b\left(Y_{1}\right), V\left(Y_{1}, Y_{1}, Z\right)\right\}\right)<-\frac{\delta\left(x_{0}\right)}{4}\right\}
\end{aligned}
$$

In words: $\mathcal{Z}\left(x_{0}\right)$ is the set of the $n-2$ lowest signals at which the bidder expects a loss of at least $\delta\left(x_{0}\right) / 4$ if he wins the auction. In this event he will not revise his 1-st round bid $b\left(x_{0}\right)$, resp. $b\left(x_{0}-\varepsilon\right)$. If in addition $x_{0}-\varepsilon<Y_{1}<x_{0}$ the bidder loses the auction and thus avoids the loss if his 1 -st round bid is $b\left(x_{0}-\varepsilon\right)$ rather than $b\left(x_{0}\right)$.

Therefore, the increment in payoff in event $A_{1}$, denoted by $\Delta_{1}$, is at 
least

$$
\begin{aligned}
\Delta_{1} & =\frac{\delta\left(x_{0}\right)}{4} \operatorname{Pr}\left\{Z\left(x_{0}\right) \cap\left\{x_{0}-\varepsilon<Y_{1}<x_{0}\right\}\right\} \\
& =\frac{\delta\left(x_{0}\right)}{4} \operatorname{Pr}\left\{Z\left(x_{0}\right) \mid x_{0}-\varepsilon<Y_{1}<x_{0}\right\} \operatorname{Pr}\left\{x_{0}-\varepsilon<Y_{1}<x_{0}\right\} \\
& \geq \frac{\delta\left(x_{0}\right)}{4} \underline{c} \varepsilon \operatorname{Pr}\left\{b\left(Y_{1}\right)-V\left(x_{0}, Y_{1}, Z\right)>\frac{\delta\left(x_{0}\right)}{4} \mid x_{0}-\varepsilon<Y_{1}<x_{0}\right\}
\end{aligned}
$$

To evaluate the probability in the last expression use the uniform continuity of $\delta$ and of $V$ and choose $\varepsilon>0$ such that

$$
x_{0}-\varepsilon<y_{1}<x_{0} \Longrightarrow\left\{\begin{array}{l}
\left|V\left(x_{0}, y_{1}, z\right)-V\left(y_{1}, y_{1}, z\right)\right| \leq \frac{\delta\left(x_{0}\right)}{8} \quad \forall z \\
\text { and } \delta\left(y_{1}\right) \geq \frac{\delta\left(x_{0}\right)}{2} .
\end{array}\right.
$$

Choose $\eta>0$ such that $\forall z ;|z|<\eta$ and $\forall y_{1}$

$$
\left|V\left(x_{0}, y_{1}, z\right)-V\left(y_{1}, y_{1}, 0\right)\right| \leq \frac{\delta\left(x_{0}\right.}{8} \text {. }
$$

(Note that $\eta$ does not depend on $\varepsilon$.) Then $\forall z ;|z|<\eta$ and $x_{0}-\varepsilon<y_{1}<x_{0}$ we have

$$
\begin{aligned}
b\left(y_{1}\right)-V\left(x_{0}, y_{1}, z\right)= & V\left(y_{1}, y_{1}, 0\right)+\delta\left(y_{1}\right)-V\left(x_{0}, y_{1}, z\right) \\
\geq & \frac{\delta\left(x_{0}\right)}{2}-\left|V\left(x_{0}, y_{1}, z\right)-V\left(y_{1}, y_{1}, 0\right)\right| \\
\geq & \frac{\delta\left(x_{0}\right)}{2}-\left|V\left(x_{0}, y_{1}, z\right)-V\left(y_{1}, y_{1}, z\right)\right| \\
& -\left|V\left(y_{1}, y_{1}, z\right)-V\left(y_{1}, y_{1}, 0\right)\right| \\
\geq & \frac{\delta\left(x_{0}\right)}{4} .
\end{aligned}
$$

It follows that

$$
\begin{aligned}
\operatorname{Pr}\left\{b\left(Y_{1}\right)-V\left(x_{0}, Y_{1}, Z\right)\right. & \left.>\frac{\delta\left(x_{0}\right)}{4} \mid x_{0}-\varepsilon<Y_{1}<x_{0}\right\} \\
& \geq \operatorname{Pr}\{|Z| \leq \eta\},
\end{aligned}
$$

and hence

$$
\begin{aligned}
\Delta_{1} & \geq(\underline{c} \eta)^{n-2} \underline{c} \varepsilon \frac{\delta\left(x_{0}\right.}{4} \\
& =c \delta\left(x_{0}\right) \varepsilon,
\end{aligned}
$$


for some constant $c>0$.

To assess the effect of the deviation from $b\left(x_{0}\right)$ to $b\left(x_{0}-\varepsilon\right)$ in event $A_{2}$ let $L:=\max _{x}|V(x, y, z)|$. Then, clearly, the payoff increment in this event, denoted by $\Delta_{2}$, satisfies

$$
\Delta_{2} \geq-L \operatorname{Pr}\left\{A_{2}\right\} \geq-L \bar{c} \varepsilon^{2} .
$$

We conclude that

$$
\pi\left(x_{0}, x_{0}-\varepsilon\right)-\pi\left(x_{0}, x_{0}\right) \geq c \delta\left(x_{0}\right) \varepsilon-L \bar{c} \varepsilon^{2},
$$

which is positive for $\varepsilon$ sufficiently small, unless $\delta\left(x_{0}\right)=0$. This completes the proof.

\section{References}

Caffarelli, F. V. (1997). Auction mechanisms for privatization: The ENI group multiple round auction procedure. Working paper, CORIPE, Torino.

Fishman, M. J. (1988). A theory of preemptive takeover bidding. Rand Journal of Economics, 19:88-101.

Landsberger, M., Rubinstein, J., Wolfstetter, E., and Zamir, S. (1997). Firstprice auctions when the ranking of valuations is common knowledge. Discussion paper, Center for Rationality, Hebrew University, Jerusalem.

Milgrom, P. and Weber, R. J. (1982). A theory of auctions and competitive bidding. Econometrica, 50:1089-1122. 